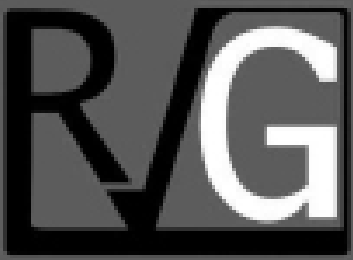

Año 24 No. 88

Octubre - Diciembre 2019

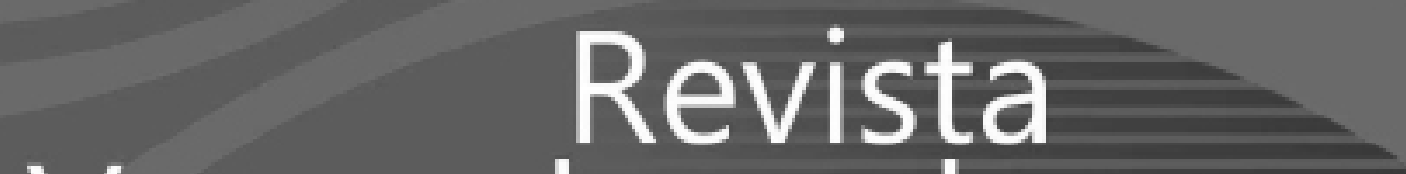

Venezolana de

verencla

UNIVERSIDAD DEL ZULIA (LUZ)

Facultad de Ciencias Económicas y Sociales

Centro de Estudios de la Empresa 


\title{
Técnicas de comercialización y diversificación de cultivos para exportación en el sector agroalimentario en México
}

\author{
Esquivel, Francisco Alonso ${ }^{1}$ \\ García Sandoval, Jesús Roberto² \\ Aldape Ballesteros, Luis Alberto ${ }^{3}$
}

\section{Resumen}

La variedad de semillas que se pueden producir en la ciudad de Valle Hermoso, Tamaulipas, México condicionan la búsqueda de nuevos espacios territoriales para su producción, la misma se basa en nuevas técnicas de recolección de datos basados en el análisis de suelos que permite determinar los nutrientes con los que cuenta la tierra, y que pueden soportar una gran variedad de tipos de cultivos. El objetivo de esta investigación es estudiar las técnicas de comercialización y diversificación de cultivos para exportación en el sector agroalimentario en México. La metodología usada se refirió a un estudio descriptivo de campo. Los resultados dan cuenta de una diversidad de técnicas de comercialización y la existencia de una diversificación de cultivos que están vinculados a los actores que intervienen en el proceso de producción. Se concluye que en México existe una política definida por el propio Estado que define técnicas de comercialización específicas para el sector agroalimentario.

Palabras clave: comercialización; cultivos; diversificación; exportación; distribución; técnicas.

Recibido: 20-03-19

Aceptado: 19-06-19

1 Universidad Autónoma de Tamaulipas, México aesquivel@docentes.uat.edu.mxOrcid ID 00000002-4985-570X

2 Universidad Autónoma de Tamaulipas, México, jrgarcia@docentes.uat.edu.mxOrcid ID 00000003-3770-1620.

3 Universidad Autónoma de Tamaulipas, México laldape@uat.edu.mxOrcid ID 0000-0001-7904$648 \mathrm{X}$ 


\title{
Marketing and diversification techniques for export crops in the agri-food sector in Mexico
}

\begin{abstract}
The variety of seeds that can be produced in the city of Valle Hermoso, Tamaulipas, Mexico condition the search for new territorial spaces for its production, it is based on new data collection techniques based on soil analysis that allows determining the nutrients that the earth has, and that can withstand a wide variety of crop types. The objective of this research is to study the techniques of marketing and diversification of crops for export in the agri-food sector in Mexico. The methodology used referred to a descriptive field study. The results show a diversity of marketing techniques and the existence of a diversification of crops that are linked to the actors involved in the production process. It is concluded that in Mexico there is a policy defined by the State itself that defines specific marketing techniques for the agri-food sector.
\end{abstract}

Keywords: Marketing; crops; diversification; export; distribution; techniques.

\section{Introducción}

En América Latina el sector agroalimentario es de relevancia significativa, fundamentalmente la producción alimentaria, tal como lo indica la Organización de las Naciones Unidas para la Alimentación y la Agricultura (2019), "América Latina y el Caribe es una región productora y exportadora de alimentos, su riqueza natural, la industria agrícola y el sector de agricultura familiar es fundamental para la seguridad alimentaria de su población", en consecuencia "la región produce suficientes alimentos para satisfacer las necesidades de todos sus habitantes. El problema central del hambre en la región no es la falta de alimentos, sino las dificultades que los más pobres enfrentan para poder acceder a ellos.", aún en este sector existen grandes diferencias, ya que la producción agroalimentaria puede ser natural u orgánica o mediante productos químicos.

El objetivo de esta investigación es estudiar las técnicas de comercialización y diversificación de cultivos para exportación en el sector agroalimentario en México, específicamente se abordan las técnicas de comercialización y diversificación de cultivos utilizada por los empresarios para exportar sus productos agrícolas y los apoyos de tipo financiero que reciben.

La metodología utilizada es una investigación es de tipo cualitativa de campo mediante encuestas, entrevistas a profundidad, muestreo de expertos, sistemático, por cuotas, de casos típicos, de máxima variación, homogéneos, en cadena, intencional y probabilístico. Se realizó una investigación entre los cuatro módulos de riego de la región noreste de Tamaulipas que comprenden más de treinta mil productores, de los cuales por cuestiones de seguridad de 
la región se negaron en su gran mayoría a facilitar parte de su terreno, del cual se necesitaban diez por dos metros para realizar una prueba de siete cultivos, que fueron okra, melón, sandía, frijol soya, calabaza y nopal, entre los que se lograron determinar que todos ellos son factibles de producir, ya que se lograron los cultivos.

La investigación comprendió entrevistas abiertas y cerradas a ochenta productores socios de una bodega que apoyó en la realización de unos estudios de análisis de suelos para determinar la razón por la cual obtenían el rendimiento registrado mediante el ingeniero agrónomo que brinda apoyo a los productores clientes.

Se logró detectar una bodega perteneciente al módulo de riego 2-II concentrada en el ejido Aquiles Serdán denominado AGRODECA quienes brindaron el apoyo de su personal para determinar los productores factibles para muestrear sus predios y determinar el nivel de calidad de suelo basado en un estudio. En un total de ochenta productores se detectó que el $95 \%$ no hacían sus análisis de suelos, por lo que, en ocasiones invertían y dosificaban cantidades mayores o menores de las requeridas para sus suelos. Se les brindó muestras de análisis de suelos gratuitas como parte del proyecto, y se esperan mejores rendimientos en la cosecha posterior a este proyecto

\section{Técnicas de comercialización: Nuevos modos en el sector agroalimentario}

La comercialización de los productos agroalimentarios atiende a una gran gama de posibilidades derivado de la diversificación de cultivos y tipos de preparación para su cosecha, preparación y comercialización que atienden al tipo de producto y de método de procesamiento para determinar su formato de tipo de comercialización.

Entonces se considera que, en una tierra de la región conocida como predio, y que se cuentan para cultivar, se pueden plantar varios tipos de semillas, y la oportunidad de trascender al entorno actual en donde se vende solo a granel, que sin duda alguna es muy viable para un variado tipo de procesamiento, comercialización y distribución, no se han considerado por el momento otras vías de venta sino que por cosecha en bodegas o a particulares quienes reciben los camiones de los productores y que posteriormente revenden para su procesamiento. Con esta investigación se da la pertinencia de indagar los tipos de cultivos pertinentes, así como las formas de procesamiento y comercialización y distribución de los cultivos pertinentes de la región. Pero para la comercialización se requiere una investigación previa de clientes potenciales, así como los requerimientos para su procesamiento, almacenaje y embalaje.

En la actualidad existen una gran variedad de apoyos para exportación de diversos productos y en Internet se pueden encontrar una gran gama de documentos que permitirán a los productores de ciudades pequeñas acceder a comercializadores y distribuidores, así como también existen eventos a nivel mundial de todas partes del mundo en donde en algunos casos tienen su streaming en vivo y en otros se requiere la presencia para ver todo lo que en ello se ofrece.

En las comunidades pequeñas con productores se requiere ahorrar para asistir a dichos eventos para 

agroalimentario

Esquivel, Francisco Alonso; García Sandoval, Jesús Roberto y Aldape Ballesteros, Luis Alberto

poder encontrar y ver de viva voz a los proveedores, distribuidores, comercializadoras e incluso proveedores de diversas áreas e incluso productores que asisten desde muy diversas partes de la república mexicana y del mundo.

Para ello los productores del agro ya cuentan con una gran variedad de eventos relacionados a productos procesados, así como de productores y empresas relacionadas al sector agroalimentario y que conlleva a la reunión de un sinnúmero de clientes potenciales, distribuidores, agencias aduanales, comercializadoras, expertos en el cuidado de semillas, cultivos, invernaderos, sistemas para el control de plagas, drones especializados en monitoreo de tierras con sus productos y sus nutrientes, empresas especializadas en el análisis de suelos, entre otras muchas variedades de empresas que apoyan a este sector incluso en maquinaria y equipo para el proceso de la siembra y procesamiento de productos.

Esquivel (2018) menciona algunos eventos recomendados para darle seguimiento "México alimentaria 2018 foodshow, Expo Agroalimentario Guanajuato 2018, Ferias Alimentarias en Estados Unidos, USA, Ferias Alimentarias en México", eventos que sin duda alguna apoyan a los productos agroalimentarios que entre otros que se dan en el mundo deben ser considerados para asistir. Es por ello que los productores asisten a estas reuniones, podrían beneficiarse con técnicas nuevas e innovadoras que se exponen en dichos eventos, así como de contactos de apoyo para sus procesos productivos.

Por otro lado, existe la vertiente de comercializar los productos de muy variadas formas, tal es el caso, como ejemplo el limón, que puede venderse a granel, pero también puede procesarse para usarse en una gran variedad de productos desde, detergentes, aromatizantes, gel, shampoo, jabón, chicle o goma de mascar y así podríamos continuar con productos que se pueden ver ya en centros comerciales, farmacias, etc., entre otras que venden productos variados. Con ello se expresa que los productores podrían intentar de penetrar nuevos nichos de mercados, dependiendo del procesamiento que se pueda dar al producto que no sea necesariamente la venta a granel.

De acuerdo con Esquivel (2018:568), existen algunas técnicas de procesamiento y comercialización:

"existen muchos otros productores

y grandes compañías que no se ciegan a la venta a granel, sino que comercializan sus productos para su venta en almíbar, jugo, concentrado, encapsulado, fruto seco, cristalizado, enlatado, escabeche, deshidratado, néctar, natural, crema, a granel, helado, rajas, rodajas, cóctel, yogurt, crema, ensalada, suplemento alimenticio, orgánico, malteada, champú, set de ciertas piezas en sobre, en plástico duro, aromatizante para spray, jabón en barra, jabón corporal líquido, jabón líquido para manos entre otras formas muy variadas en donde aplica el uso de productos agroalimentarios que no necesariamente son de consumo en formas variadas sino que su esencia y aroma también es factible de ser utilizado, para ello proveedores, distribuidores y comercializadoras".

Los productos agroalimentarios cuentan hoy en día con una gran gama de posibilidades de procesamiento y comercialización, que no solo se limita a la venta a granel y que aun siendo a granel puede ser a empresas que procesan, comercializan y distribuyen los productos; en las diversas formas 
mencionadas entre otras muchas, puede crearse un convenio de colaboración con algunas de las marcas o empresas comercializadoras como las que entre otras muchas otras. Con esto se debe considerar el contactar a las empresas especializadas para que sea utilizado para el procesamiento especializado. Para ello ya se cuenta con empresas que compran a granel los frutos o productos agroalimentarios, y que procesan o incluso venden a granel en gran escala a ciertos centros comerciales de marca y que distribuyen a nivel nacional e internacional.

Entre algunas empresas que se dedican a procesar y comercializar los productos agroalimentarios que se derivan de compra a productores directos o comercializadoras especializadas se encuentran una gran gama de empresas que diversifican la variedad de productos y formas de transformación de un producto natural para captar su esencia o aroma y así transformarlo en un producto para el mercado. Por ello Esquivel (2018) comenta que algunas empresas que se dedican a la venta, distribución y comercialización de productos agroalimentarios y transforman en otra variedad procesada para su puesta a la mano del consumidor, se encuentran: El Sureño, Desierto, Agros, Mery Peppers, Heb, Gasperin, Mañanita, Golden Great Brand, Grupo Cabello entre otras. Los requerimientos se orientan a que sean productos diversos con formatos de venta, comercialización y distribución, como ejemplo, el limón como tal, no solo puede ser comercializado a granel, sino como aroma o esencia para perfumes, desodorantes, entre los ya mencionados.

Con esto se considera que, que los productores necesitan determinar el cultivo y con ello investigar todas las posibilidades en la gama de comercialización pertinentes para dicha semilla o producto que podría derivar a la diversificación de cultivos, venta comercialización y distribución del mismo; en consecuencia se necesita la determinación de crear un estudio de mercado para ver las posibilidades existentes que ya son mayores a las se tenían en décadas anteriores dentro de la zona de estudio en esta investigación.

Por tanto, se pueden utilizar páginas web 0 aplicaciones de dispositivos móviles, tal como Esquivel et al (2018:578) menciona:

Una de las formas de dar seguimiento a los productos y apoyos es sin duda alguna a través de aplicaciones para dispositivos móviles...para lo cual primero necesitamos determinar el tipo de aplicaciones basado en la usabilidad que deseamos, entre las cuales podrían ser por suelos, aguas, malezas, producto, maquinaria, precios, proveedores, importadores y exportadores, e incluso de apoyos y convocatorias entre toda la gama de posibilidades tal como la app Agrimercante produce (s.f.) que podría apoyarnos a la toma de decisiones sobre la comercialización y producción.

$Y$ que como ya es sabido, las aplicaciones móviles, así como el uso de redes sociales son hoy en día una pauta para tener información a la mano en cualquier momento.

Por otro lado, el Programa Nacional de Financiamiento al Microempresario (2017) en su convocatoria "Para impulsar la adquisición de conocimiento de los y las microempresarias, en el PRONAFIM emitimos convocatorias para la incubación de actividades y proyectos productivos", da soporte y ayuda a los micro empresarios que deseen incubar sus ideas de negocios entre los cuales existirían una gran variedad de tipo de producto para venta derivado de la gama 
Técnicas de comercialización y diversificación de cultivos para exportación en el sector agroalimentario

Esquivel, Francisco Alonso; García Sandoval, Jesús Roberto y Aldape Ballesteros, Luis Alberto

de posibilidades de comercialización mencionadas y que los productores podrían iniciar si lo desean, y que al final de todo es cuestión de querer ya que tienen las tierras y los insumos para producir varios cultivos, y que en determinado caso solo necesitan la forma de comercializar para procesarlos y emprender su propio negocio.

Ya por su parte Esquivel, F.A. (2018:229). Argumenta que "Existe una inquietud de diversificar el cultivo y aspirar a exportar no sólo a Estados Unidos, sino también a América Latina y Europa, los productos actuales o nuevos en caso de que sean factibles de concebir." Y para ello se tiene que determinar el tipo de producto que se desea comercializar y hacer un verdadero estudio de mercado, para determinar los apoyos que se deben de conseguir y con ello iniciar la odisea. Pero también comenta que "Ahora bien, existe mucho interés en diversificar el cultivo, pero no la certidumbre de poder comercializarlo o exportarlo a algunas otras zonas o regiones nacionales e internacionales", por esta justa razón que se deben determinar los principales recursos mediante las factibilidades técnicas, operativas y financieras mediante un plan de negocios y así apoyarse lo más posible con las estrategias de negocios posibles para determinar las necesidades y posibilidades existentes, ya sea con recursos propios o atendiendo a diversos organismos para conseguir los apoyos necesarios.

\section{Diversificación de cultivos: Algunos procedimientos necesarios}

Existen variadas formas de comercialización de frutos, la gama de posibilidades de cada producto y sus derivados es de gran diversidad, debido a que un fruto puede ser aprovechado de muchas maneras.

La empresa PRODES (2017) promotora de deshidratados se encarga de procesar los productos de una gran variedad de productores tales como frutas deshidratadas, para ello mencionan que los productos deshidratados son obtenidos mediante la infusión con azúcar y secado por calor. Se considera que, la gran posibilidad de producir el producto que nuestro cliente requiere exista en diversas presentaciones y variedades de consumo para que los productos sean realmente atractivos para los consumidores finales, entre los productos que reciben son: fresa, melón, plátano, frambuesa, manzana, piña, mango, papaya, durazno y coco.

De otra forma la PROFECO (2017). En informe realizado menciona que entre otra gran variedad de recetas, también muestra la forma de "Frutas deshidratadas", y para ello entre otra información muestra que la deshidratación es uno de los métodos más rudimentarios y antiguos utilizados por el hombre para conservar alimentos, ya que al retirarles al máximo el contenido de agua se evita el desarrollo de microorganismos.

Por su parte PROFECO (2018), indica las "Tecnologías Domésticas Profeco" y en su apartado del "En el almacén" menciona algunas formas de crear productos: aderezos, cárnicos y embutidos, consejos prácticos, confitería, congelación, conserva de frutas y verduras, instantáneos, lácteos, panificación, pescados y mariscos, procesos de esterilización, procedimientos y técnicas, productos de uso en el hogar y productos de uso personal. 
Es apremiante investigar alternativas productivas que apoyen a la economía familiar durante el ciclo de producción de aquellos productos como sorgo y maíz los cuales el tiempo de la siembra a la cosecha es de aproximadamente de 80 a 150 días. Estas alternativas deben favorecer la economía de las familias que económicamente dependen de la agricultura. La diversificación de cultivos es una alternativa para resolver problemáticas socioeconómicas que aquejan a los productores agrícolas; esta alternativa consiste en cultivar productos alimenticios de corto plazo los cuales auxilien durante la producción de los productos como el sorgo y el maíz. La diversificación de cultivos es una opción sólida que consciente mejorar la inversión del productor, requiere menor cantidad de tierra utilizada, así como gastos mínimos en su producción.

La diversificación de cultivos tiende a comprender ciertos pasos para que esta pueda ser exitosa esta diversificación se puede compartir en los ciclos de siembra del sorgo y el maíz utilizando el mismo espacio y los mismos nutrientes, así como el mismo trabajo; por ello se hace necesario destacar las siguientes recomendaciones:

- Elección de cultivo que se ajuste a la región

- Cercanía o acceso a tomas de agua

- Que los requerimientos de agua sean similares.

- Las distancias entre las plantas deben considerarse para el proceso de trabajo o mantenimiento.

- Considerar los cultivos sean resistentes a los insecticidas 0 plaguicidas ya sean naturales o químicos.

- Considerar la siembra de leguminosas por los nutrientes que otorgan a las tierras.

Estas entre otras tantas recomendaciones que pueden variar de acuerdo con el contexto, deben considerarse o tomarse en cuenta al momento de diversificar o alternar cultivos.

\section{Instituciones que promueven el apoyo para exportar en México}

Se precisan las instituciones que promueven apoyo para los procesos de exportación en México

\subsection{Instituciones Federales}

ProMéxico (2017) brinda una gran variedad de asesorías y apoyos para las personas que desean exportar y comercializar, entre los servicios que ofrece en línea se considera que, Asesoría en empaque y embalaje, Asesoría legal internacional, Asesoría para la formación de consorcios de exportación (REDEX), Asesoría técnica en procesos productivos, Centros de distribución, Certificaciones internacionales, Consultoría para registro de marca internacional (IMPI), Desarrollo de estrategia de e-commerce y marketing digital, Ferias tipo B, Ferias tipo C, Formación de ejecutivos en comercio exterior, Misiones estratégicas de comercio (mexicanos), Misiones estratégicas de comercio (extranjeros), Strategic Trade Missions (foreigners), Programa de promoción internacional: Gerente de redes de exportación (REDEX), Agenda de negocios, Alianzas estratégicas, Asesoría especializada, Desarrollo de un proyecto en comercio exterior, Ferias tipo A, Inteligencia técnica para exportadores, Oferta exportable en stand institucional, Pop 
Técnicas de comercialización y diversificación de cultivos para exportación en el sector agroalimentario

Esquivel, Francisco Alonso; García Sandoval, Jesús Roberto y Aldape Ballesteros, Luis Alberto

up store / showroom Pop up store / showroom, Presentación de productos: degustación y/o cata, Programa de capacitación en comercio internacional, Publicidad en medios.

Aunado a ello ProMéxico (s.f.) tiene un "DIEX - DIRECTORIO DE EXPORTADORES" que ayuda a localizar a proveedores o clientes dependiendo de la naturaleza del contacto y que pueden apoyar al productor con los requisitos a los países que desee exportar.

Por otro lado, en su apartado de "Preguntas frecuentes" para exportaciones, brindará una gran gama de posibilidades para determinar si está o no preparado, así como un gran contenido de preguntas básicas que ayudarán a resolver los requisitos para exportar por tipo de producto.

Continúa mencionando en su apartado de "Pasos para exportar" brinda una adecuada ayuda sobre este tópico con los temas bien descritos tales como: a) Consideraciones para exportar; b) Preparación para exportar; c) Documentos y requisitos generales para exportar; d) Cadena de suministros y logística de exportación.

Mientras tanto que MexBest (2018)"es un proyecto creado por SAGARPA para presentar y promover los productos agroalimentarios del campo mexicano con calidad de exportación, diversidad y sabor que nos permiten llegar a cualquier parte del mundo" pero también cuenta con una gran gama de servicios para la comunidad tales como para el Productor Mexicano: "Encuentra toda la información que necesitas saber para poder formar parte del Directorio Mexbest, y cuenta con grandes beneficios de negocio al poder exportar tus productos a nivel internacional con el respaldo de SAGARPA."

Certificados: "En MexBest encontrarás los mejores productos agroalimentarios mexicanos, con la seguridad certificada de que tendrás en tus manos productos bajo los más altos estándares internacionales."

Directorio de Agroexportadores: "Englobamos el esfuerzo de más de 1200 exportadores mexicanos que comercializan diversos productos agroalimentarios de la mejor calidad, y que cuentan con las principales certificaciones para que el mundo los conozca." Entre otras múltiples y muy variadas opciones para que el productor se apoye al momento de decidir exportar sus productos.

\subsection{Instituciones privadas}

La plataforma de Yo Exporto (2016) es "Una plataforma en línea para aprender a exportar. Te llevamos de la mano para internacionalizar tus productos" y que pretende apoyar a las personas a su proceso de exportación mediante "El curso de 16 temas y 50 horas que te llevarán a exportar. A través de cada uno de los temas, podrás adquirir todos los conocimientos necesarios para entender y ejecutar el proceso de exportación" y con ello poder exportar en caso de desear iniciar con el proceso de aprendizaje de exportar, aunque existen otras plataformas del gobierno federal gratuitas para la asesoría.

Pero también se encuentran plataformas que ayudan a conseguir apoyos de fondos convocatorias Federales, y de acuerdo a Massive Dynamics (s.f.) tal como Prana México que ofrece "Cotizar Mi Solicitud de Apoyo. Encuentra El Programa Justo Y Cotiza. (SAGARPA, INADEM, CONACYT y Más)" entre otros como "INAES, SEDESOL, SEMANART" y muchos más. En los que ellos tienen experiencia para 
descargar fondos federales.

Esto conlleva a que se requiere solo tener la voluntad de querer hacer las cosas para así buscar la plataforma que apoye a descargar recursos o a atender las convocatorias de manera personal y correr el riesgo de no tener soporte para realizar los documentos o simplemente recibir la asesoría de plataformas que cuentan con una vasta experiencia en descargar fondos diversos para proyectos emprendedores o de micro y pequeñas empresas.

Por su parte, Produce Marketing Association (2017) nos indica "cómo exportar frutas y vegetales frescos de México a estados unidos" y también comenta que:

Las frutas y los vegetales frescos que se importan a EE. UU., deben cumplir las mismas reglamentaciones que aquellos que se producen en EE. UU., además de que los productos deben ser inocuos y no contener ingredientes prohibidos. El etiquetado y el envasado también son componentes críticos.

Es por esto que se requiere conocer los términos en los que se realizarán los productos y las normas que deben cumplir para pretender exportar a otros países. En este sentido existen diversos actores que intervienen en la diversificación y en la comercialización de cultivos; existe una definición de la política de parte del Estado mexicano.

\section{c) Actores regionales que apoyan al sector agroalimentario}

Son diversos los actores que participan en el proceso de comercialización y diversificación de los cultivos, entre ellos se encuentran, la Secretaría de Agricultura, Ganadería,
Desarrollo Rural, Pesca y Alimentación, que tiene varios programas por nombrar algunos el Programas SAGARPA 2018, brinda apoyos para semillas mejoradas, fertilizantes, maquinaría, capacitación y entre otros.

El Programa de Fomento a la Agricultura, se orienta al componente de Energías Renovables, Componente PROAGRO Productivo, Componente de Mejoramiento Productivo de Suelo y Agua, Componente de Investigación, Innovación y Desarrollo Tecnológico Agrícola, Componente de Estrategias Integrales de Política Pública Agrícola, Componente de Capitalización Productiva Agrícola.

Otro programa que resalta y está, orientado a los productores es el denominado Programa de Apoyos a Pequeños Productores, comprende el Proyecto de Seguridad Alimentaria para Zonas Rurales, Componente Infraestructura Productiva para el Aprovechamiento Sustentable del Suelo y Agua (IPASSA) (Ejecución Nacional).

Proyectos Productivos (FAPPA), Programa de Apoyos para Productores de Maíz y Frijol (PIMAF), Componente PROCAFÉ e Impulso Productivo al Café, Componente Fortalecimiento a Organizaciones Rurales, Componente el Campo en Nuestras Manos, Programa de Desarrollo de las Zona Áridas (PRODEZA), Componente Atención a Siniestros Agropecuarios, Componente Arráigate Joven - Impulso Emprendedor, Componente Extensionismo, Desarrollo de Capacidades y Asociatividad Productiva.

$\begin{array}{ccc} & \text { Programa de } & \text { Productividad } \\ \text { y Competitividad: } & \text { Componente }\end{array}$
Riesgo Compartido, Componente de Fortalecimiento a la Cadena Productiva, Componente de Desarrollo Productivo del Sur Sureste y Zonas Económicas 
Técnicas de comercialización y diversificación de cultivos para exportación en el sector agroalimentario

Esquivel, Francisco Alonso; García Sandoval, Jesús Roberto y Aldape Ballesteros, Luis Alberto

Especiales, Componente de Certificación y Normalización Agroalimentaria, Componente de Activos Productivos y Agrologística, Componente Acceso al Financiamiento.

Programa de Sanidad e Inocuidad Agroalimentaria: Componente Inocuidad Agroalimentaria, Acuícola y Pesquera, Componente Campañas Fitozoosanitarias, Componente Inspección y Vigilancia Epidemiológica de Plagas y Enfermedades No Cuarentenarias, Componente Vigilancia Epidemiológica, de Plagas y Enfermedades Cuarentenarias.

Programa de Apoyos a la Comercialización: Componente Promoción Comercial y Fomento a las Exportaciones, Componente Incentivos a la Comercialización.

Todos estos programas mencionados previamente estuvieron y están encaminados a favorecer el proceso de producción y comercialización de pequeños agricultores y ejidatarios en México, cada uno de ellos con características y funciones distintas, pero orientadas a la mejora del proceso productivo y por tanto a mejorar la economía de las familias que dependen directa o indirectamente de la agricultura en las diversas regiones del país reduciendo la pobreza y otorgando mejores oportunidades de crecimiento y competitividad. Los programas descritos al menos en la teoría tratan de modernizar el sector agrícola para dejar atrás la brecha tan marcada que existe en este mismo sector productivo y de gran trascendencia para la economía del país.

\section{La experiencia del estado de Tamaulipas}

En México la producción agropecuaria su dinámica demuestra un avance importante por los actores que se vinculan para su desarrollo, en ciudad de Valle Hermoso, ubicada en el Estado de Tamaulipas en México, existen organismos que son determinantes en la publicación y asesoría de información para los productores, tal es el caso que la Unión Agrícola Regional del Norte de Tamaulipas (UARNT), Secretaría de Agricultura y Desarrollo Rural (SAGARPA), Centro de Apoyo al Desarrollo Rural (CADER), III Valle Hermoso, Servicio de Información Agroalimentaria y Pesquera (SIAP), las oficinas, portales de información o ventanillas que atienden a convocatorias del gobierno federal, ponen a disposición de la sociedad en general información variada y útil para ser considerada al momento de determinar las necesidades o requerimientos de los productores, y hasta cuentan con aplicaciones para dispositivos móviles tal como el Atlas agroalimentario 2017 versión 2.0 en dónde se puede observar en las consultas de información todo lo relacionado a las producciones agrícolas por producto o por Estado, en las que se observan detalles técnicos y ventas nacionales y el lugar que ocupa cada producto a nivel nacional e internacional, así como las ventas.

La Secretaría de Desarrollo Rural del Estado de Tamaulipas (2018) mencionó que para "El ciclo otoño invierno es uno de los más importantes ya que se siembran la mayor cantidad de hectáreas, en el ciclo 2015-2016 se sembraron 917,789 hectáreas, los principales cultivos de este ciclo son: Sorgo grano, Maíz grano, Cártamo, Canola, Algodón hueso, Cebolla, Okra, Arroz palay, Trigo y Girasol."

En términos generales se considera que, que en las comunidades pequeñas como el caso de la Ciudad de Valle 
Hermoso en el Estado de Tamaulipas y la región que comprende municipios pequeños mencionados anteriormente, no existe la posibilidad, por el momento, de que trasciendan a los cultivos de Maíz, y Sorgo que principalmente se producen en esta región. Si bien es cierto que existen múltiples convocatorias, eventos y apoyos para el sector agroalimentario tal como la convocatoria de Walmart "Convocatoria Pequeño Productor 2018" que al final de la convocatoria menciona:

Los siguientes 50 finalistas iniciarán el proceso de estudio socioeconómico mediante el cual se verificará que sean Pequeños Productores de acuerdo a la descripción de la convocatoria. En caso de que uno o varios de los participantes no cumplan los requisitos, el proveedor con la siguiente mejor calificación ocupará su lugar.

Convocatoria es específicamente para que los productores puedan comercializar sus productos si cumplen con los requisitos mencionados, y que, pueden ser causa de comercialización para los pequeños productores de la región si así desean hacerlo y participar en dicha convocatoria siempre y cuando estén preparados y la convocatoria la refieren a a) Pequeños Productores con alguna situación de vulnerabilidad, b) Fundaciones y organizaciones que trabajen con pequeños productores $y$, c) Universidades y grupos estudiantiles que trabajen con pequeños productores

Con ello se considera que, que sí existe la posibilidad de incorporarse a la participación de este tipo de convocatorias, y lo único que hay que desear, es salir de una situación haciendo lo necesario para poder realizarlo y atender dicha convocatoria.

Para ello, en la investigación de apoyo PRODEP "Diversificación de producción de cultivos en Valle
Hermoso y factores determinantes para la exportación y financiamiento público o privado." Desde junio del 2018 se le dio a conocer a los diversos productores participantes en el proyecto y de los cuales ni uno solo consideró la idea de participar porque "eran muchos los requisitos", y si bien es cierto, las convocatorias, eventos y apoyos se abren todos y cada uno de los años, de nada servirá que existan si los productores no desean invertir tiempo, dinero y esfuerzo para salir de su propio entorno.

Por su parte Florez (2015) ya ha comentado que:

El Censo Agropecuario de 2007 también contiene el dato de las personas vinculadas a las actividades agrícolas. Así se sabe que en 2007 se contrataron alrededor de cinco millones de personas, más 3.5 millones de personas familiares del productor, con lo que hubo un total de 8.5 millones de personas en actividades relacionadas con el sector agropecuario y forestal. Para este mismo año, la ENOE reportó 6.1 millones de personas vinculadas a las actividades del sector primario de la economía.

Esta aseveración no implica que todos los productores estén vinculados al sistema de exportaciones y comercialización de productos agroalimentarios 0 que sea de trascendencia. En el año 2018 está fuera de contexto, en razón de los procesos de exportación y comercialización de diversas maneras, con respecto al productor que desea hacer e investigar el cómo lograrlo, solicitando apoyo a los diferentes organismos que ya existen hoy en día Tal como ProMéxico que ofrece entre sus múltiples servicios en Directorio de exportadores (DIEX) y es de acceso libre, así como la Secretaría de Economía entre otros muy variados 

agroalimentario

Esquivel, Francisco Alonso; García Sandoval, Jesús Roberto y Aldape Ballesteros, Luis Alberto

organismos y empresas que podrían apoyar al sector agroalimentario.

Por ello se necesita la vinculación con organismos federales, estatales, locales y privados para que el sector agroalimentario, logre resultados significativos, tal es el caso de comunidades pequeñas que logran acrecentar su nivel de producción tal como lo menciona el portal de Municipios Puebla, Rojas (2018), que a EEUU se exporta la mitad de la producción de nopal de Tlaxcalancingo, esto evidencia que a pesar de las condiciones del municipio han buscado trascender sus ventas $y$ han logrado penetrar nichos nuevos de mercado, de acuerdo con la noticia que se ha dado a conocer a nivel nacional en diversos medios.

En la actualidad, la región donde se encuentra la ciudad de Valle Hermoso, existe una gran mayoría de productores que siembre Maíz y Sorgo únicamente. La tierra puede producir otros cultivos, por alguna razón se ha venido ejerciendo estos dos cultivos de manera sistemática, sin embargo, las tierras y la zona de esta región, puedan soportar otros cultivos que son mejor pagados tal como el frijol soya. Derivado de ello y en virtud de entrevistas con productores que están preocupados por el bajo precio del maíz y sorgo, pero que no han estudiado las formas en que se pueda gestar el cambio de producto cultivado a cualquier otro. Se ha mostrado una gran preocupación, de tal forma que se necesitan más investigaciones de apoyo en este campo del conocimiento para brindar una orientación a los productores y determinar qué otros cultivos podrían incursionarse en estas tierras.

\section{Reflexiones finales}

Con esta gran gama de información podríamos considerar que cualquier persona puede iniciar su propio negocio ya sea por cuenta propia o con apoyos gubernamentales de convocatorias a fondo perdido o con el pago de asesoría mediante empresas expertas en brindar asesoría a productores y comerciantes.

La producción de cultivos se puede tener en grande o pequeña escala, pero lo más importante es determinar qué es lo que desea hacer y determinar el tipo de producto a cultivar, procesar, comercializar, distribuir 0 incluso exportar, si así lo desea, y que en todo caso y derivado de esta investigación se puede iniciar con productos caseros siempre y cuando se cumplan con las normas que se especifican en salubridad.

En el contexto donde se realizó la investigación la diversificación de cultivos se realiza con fines de autoconsumo o consumo familiar. No obstante que existen productos agrícolas que son aptos para el tipo de tierra, clima etc. la mayoría de los agricultores se centran en el cultivo de sorgo y maíz, principalmente por dos cuestiones: el primero por tradición familiar o tradición regional y un segundo por el desconocimiento del mercado o lugar donde comercializar su producto.

En el sector agrícola los procesos de comercialización son fundamentales para favorecer la economía del productor, sin embargo, este proceso de comercialización es el que más adolece el productor mexicano, ya que del campo a la mesa del consumidor final intervienen terceras personas que son las que mayor provecho obtienen en este proceso. Lo anterior hace necesario destacar que no solo es importante generar apoyos donde se otorguen financiamiento para modernizar los procesos de producción de cultivos, se hace necesario un proceso de 
formación y capacitación en áreas de administración y comercialización que les brinden las oportunidades de conocer los precios reales de sus productos para que su venta sea lo más digna posible y por lo tanto mejorar la economía en las zonas rurales.

\section{Referencias bibliográficas}

Bellante, L. (2017). Building the local food movement in Chiapas, Mexico: rationales, benefits, and limitations. Agriculture and human values. My ideas 34(1), 119-134.

Esquivel, Francisco Alonso. (2018), Diversificación de productos agroalimentarios y tipos de comercialización de frutos, Memorias del congreso internacional de investigación Academia Journals en ciencias y sustentabilidad 2018: Academia Journals, México.

Esquivel, Francisco Alonso. (2018), Vinculación de los sectores de apoyo al agro para mejora de rendimientos de producción, Memorias del congreso internacional de investigación Academia Journals en ciencias y sustentabilidad 2018: Academia Journals, México.

Esquivel, Francisco Alonso. (2018), Emprendimiento e innovación: Transformar desde las Instituciones de Educación Superior, Ediciones Astro Data S.A., Venezuela.

Florez Vaquiro., Nelson. (2015). Economía y trabajo en el sector agrícola. Facultad Latinoamerica de Ciencias Sociales, México

MEXBEST (2018). ¿Qué es MexBest? recuperado en: http://www.mexbest.com/index. fecha de consulta: $21 / 11 / 2018$
Promotora de deshidratos (2017). PRODES ¿Quiénes somos?, recuperado en: http://prodes.com.mx/\#nuestrosproductos. fecha de consulta: 08/02/2018

Produce Marketing Association (2017). Cómo exportar frutas y vegetales frescos de México a Estados unidos, recuperado en: https://www.pma. com/es/Content/Articles/2017/06/Como-exportar-frutas-y-vegetales-frescos-de-Mexico-a-Estados-Unidos fecha de consulta: 08/08/2018

Procuraduría General del Consumidor (2018). Tecnologías Domésticas Profeco, Conserva de frutas y verduras, recuperado en: https://www. profeco.gob.mx/tecnologias/conserva/conserva.asp fecha de consulta: $17 / 11 / 2018$

Procuraduría General del Consumidor (2017). Frutas deshidratadas, recuperado en: https://www.profeco.gob. $\mathrm{mx} /$ tecnologias/conserva/fdeshid. asp. fecha de consulta: 17/02/2018

Programa Nacional de Financiamiento al Microempresario (2017). Convocatorias PRONAFIM, recuperado en:https://www.gob.mx/ pronafim/acciones-y-programas/convocatorias-141072. fecha de consulta: $21 / 11 / 2018$

ProMéxico (2017). Directorio de exportadores, recuperado en: http://www. promexico.gob. $\mathrm{mx} / \mathrm{es} / \mathrm{mx} / \mathrm{diex}$. fecha de consulta: 21/03/2018

Rojas F., M. (2018). Exportan a EE.UU. mitad de la producción de nopal de Tlaxcalancingo, recuperado en http:// municipiospuebla.mx/nota/201706-04/san-andr\%C3\%A9s-cholula/ exportan-eeuu-mitad-de-la-producci\%C3\%B3n-de-nopal-de-tlaxcalancingo/ .fecha de consulta: 07/12/2018

Secretaría de Desarrollo Rural (2018). Agricultura, recuperado en: https:// 
Técnicas de comercialización y diversificación de cultivos para exportación en el sector agroalimentario

Esquivel, Francisco Alonso; García Sandoval, Jesús Roberto y Aldape Ballesteros, Luis Alberto

www.tamaulipas.gob.mx/desarrollorural/temas-del-sector/agricultura/. fecha de consulta: 05/11/2018

Walmart (2018). Convocatoria Pequeño Productor 2018, en: desde https:// www.walmartmexico.com/convocatoria-pequeno-productor-2017 fecha de consulta: 22/11/2018

Yoexporto (2016). ¿Qué es yo exporto?, recuperado en: http://www.yoexporto. $\mathrm{mx} /$. fecha de consulta: $15 / 10 / 2018$

- Esta obra está bajo una licencia de Creative Commons Reconocimiento-NoComercial- Compartirlgual 3.0 Unported. http://creativecommons.org/licenses/by-nc-sa/3.0/deed.es_ES 